Doi: $10.32481 /$ djph.2021.12.002

\title{
Public Health Genetics/Genomics
}

\author{
Louis E. Bartoshesky, MD, MPH, FACMG
}

Genetics, according to The American Heritage Dictionary, is that branch of biology that deals with heredity, especially the mechanisms of hereditary transmissions and the variation of hereditary characteristics among organisms. ${ }^{1}$ Genomics is the study of all of the nucleotide sequences - including structural genes, regulatory sequences and non-coding DNA segments - in the chromosomes of an organism. Genomics is a relatively new concept, as witnessed by the American College of Medical Genetics changing its name to the American College of Medical Genetics and Genomics in 2012. In general, clinical genetics deals with one person, and one gene locus, while genomics deals with all or large segments of the genome (multiple genes) and how all or various parts of the genome interact with one another and with the environment. Down syndrome is a genomic condition associated with an extra copy of 300+ genes on chromosome 21 , while cystic fibrosis is generally considered a genetic disorder associated with altered copies of genes at a single locus in a single person. (But perhaps CF could be considered genomic when multiple variants - mutations at that locus - are being studied within a population.)

Public health can be described as the science and art of preventing disease, prolonging life through the organized efforts of society, organizations, and individuals. Public Health Genetics/Genomics has been described as applying genetic and genomic information to improve public health and prevent disease. ${ }^{2}$ The core functions of public health include assessment, policy development, and assurance that all populations have access to appropriate care. Public Health genomics is applying such core functions to genomics. For example, core functions in Public Health Genomics might include evaluation of a state run newborn screening program (Assessment) suggesting changes in the panel of disorders screened for (Policy Development) and organizing an appropriate advisory board to assist in assuring the NSP program is reaching all infants (Assurance). Another example might include applying the genomics/public health core functions to a community based molecular breast cancer screening program.

\section{Public Health and Genomics}

Traditionally, preventive services have been on the forefront of Public Health activities. Three types of prevention are generally recognized: Primary - the prevention of disease; secondary reducing the spread of a disease in an individual or population; and tertiary - managing the long term impact of an ongoing disease. ${ }^{3}$ In clinical genomics, sometimes laboratory reports are uncertain and difficult to interpret. Perhaps there should be a fourth type of prevention: quaternary prevention or identifying a patient or population with an uncertain course putting them at risk for "over medicalization." A type of prevention to identify and protect those individuals and populations for whom medical intervention is likely to be a risk for more harm than good. ${ }^{4}$

\section{Status of Public Health Genomics}

Currently public health genomics is being integrated into existing paradigms for the provision of traditional public health services. We can expect continued alignment with current public health programs in the community designed to benefit the population. ${ }^{5}$ Clinical genomics is no longer considered only for "rare" conditions, but all disorders and diseases including conditions such as 
hypertension, various cancers, psychiatric disorders, diabetes etc.- have aspects relevant to Clinical Genomics. ${ }^{3}$

\section{Issues and Concerns}

There are issues and concerns around Clinical and Research Genomics. Public Health genomics is generating large amounts of data: the human genome includes up to 3.3 billion base pairs: 20,000 or so genes coding for a protein and similar numbers of genes not coding for a protein. Increasingly, sophisticated informatics will be necessary. Recently, a number of helpful clinical resources have become available, including, but not limited to:

- Gene Reviews, a comprehensive clinical resource;

- OMIM, an online catalogue of human genes and genetic disorders;

- The Genetic Alliance, a resource for consumers;

- The National Coordinating Center for Regional Networks, designed to improve access to Genomics services;

- Clingen, an NIH funded program dedicated to building an authoritative central resource that defines the clinical relevance of gene, genomes and variants; and

- Clinvar, a freely accessible public archive of reports of the relationship among human variations and phenotypes.

As genomics matures, the concept of Precision Medicine is being widely considered. Precision Medicine has been defined as "an emerging approach for disease treatment and prevention that takes into account individual variability in genes, environment and lifestyles for each person."6 More simply, it may be considered as providing the right treatments at the right time, every time, to the right person.

Direct to consumer advertising around molecular testing is widely available raising concern about appropriate interpretation of genomic information by consumers. Regulation is being discussed.

Uncertainty around molecular testing interpretation is widely recognized. Unexpected ("secondary") results are not uncommon; for example diagnostic exome sequencing (determining the sequence of all or most of the base pairs within the coding regions of the chromosomes) done on an infant with developmental delay might inadvertently uncover a mutation associated with high risk for an adult onset cardiomyopathy. How should that be handled?

Also, molecular testing for a specific disorder might result in the detection of a DNA variant (a mutation) not previously recognized as pathogenic (disease causing). These are labelled as variants of uncertain significance (VUS) and demand interpretation and discussion. Pre- and post-testing counseling by sophisticated genetics professionals such as Masters Level genetic counselors are needed.

Costs for genetics/genomics services are going down, but remain difficult for some consumers to afford and insurance coverage is inconsistent. Does this suggest problems around one of the core principles of public health: assurance; and to one of the basic principles of modern bioethics: justice? 
Furthermore, there is not widespread access to genomics services among lower middle income countries (LMIC), and, for that matter, within parts of the United States. These situations again raise the question of Justice.

Confidentiality, privacy, and strict adherence to bioethical principles are essential parts of Public Health Genomics and genetics activities.

\section{How should Public Sector genomics services be monitored?}

Translation of lab results to clinical availability and reliability of testing are topics that lend themselves to public health genomics discussions.

The heart of public health today is the recognition that health outcomes in populations and individuals are influenced by a range of social, cultural, political, economic, environmental, and behavioral factors. Genomics is being added, perhaps near the top of the list. ${ }^{5}$

\section{References}

1. The American Heritage Dictionary of the English Language. $4^{\text {th }}$ Ed. (2000). Houghton Mifflin, Boston

2. What is Public Health Genetics? (2021). https://www.phgw.org/wp-2021

3. Khoury, M. (2000). Genetics and Public Health in the 21st Century. Oxford University Press; 2000, New York

4. Burke, W., Burton, H., Hall, A. E., Karmali, M., Khoury, M. J., Knoppers, B., . . .. Zimmern, R. L., \& the Ickworth Group. (2010, December). Extending the reach of public health genomics: What should be the agenda for public health in an era of genome-based and "personalized" medicine? Genet Med, 12(12), 785-791. PubMed

5. Molster, C. M., Bowman, F. L., Bilkey, G. A., Cho, A. S., Burns, B. L., Nowak, K. J., \& Dawkins, H. J. S. (2018, September 4). The evolution of public health genomics: Exploring its past, present and future. Frontiers in Public Health, 6, 247. PubMed https://doi.org/10.3389/fpubh.2018.00247

6. Khoury, M. J. (2018, May). Precision public health: What is it? Centers for Disease Control and Prevention. Genomics and Precision Health. Retrieved from:

https://blogs.cdc.gov/genomics/2018/05/15/precision-public-health-2/

Copyright (c) 2021 Delaware Academy of Medicine / Delaware Public Health Association.

This is an Open Access article distributed under the terms of the Creative Commons Attribution Non-Commercial License (https://creativecommons.org/licenses/by-nc-nd/4.0/) which permits unrestricted non-commercial use, distribution, and reproduction in any medium, provided the original work is properly cited. 